\title{
ESA plans put solar science out of orbit
}

[MUNICH] European Solar System scientists, hit hard last year by the failure of Cluster and the Russian Mars 96 mission - are now the biggest losers in the reshuffling of the long-term space science plan of the European Space Agency (ESA).

The plan, known as Horizons 2000, was revised last week by ESA's Space Science Advisory Committee, a group of a dozen independent space scientists, to cope with budgets which are likely to be at least 25 per cent lower than planned for after 2000.

One immediate result has been the decision to drop from the schedule a planned mission to the planet Mercury. The budget cut is also likely to mean that European scientists will play a relatively minor role in the planned international missions to Mars and the Next Generation Space Telescope.

Horizons 2000 was approved by ESA in 1994 as a 20 -year space science programme, with launches of major 'cornerstone' missions every three to five years interspersed with medium-size missions. Its goal is to ensure a regular flow of results to each of Europe's space science research communities, including astrophysicists, planetologists and fundamental physicists.

In the optimistic climate of the time, the plan assumed not only index-linked growth in ESA's science budget to the end of the century but also a 5 per cent annual growth in real terms between 2001 and 2005.

But, late last year, ministers from ESA's 14 member states decided that the growth of the budget of the space science programme should no longer be increased at the rate of inflation between 1996 and 1998. In the absence of further ministerial action, the level budget will automatically continue for two more years and a real increase in budget after 2000 now seems out of the question.

Despite the new budgetary constraints, the advisory committee, whose recommendations have always been accepted by ESA, wants to maintain all the scientific aims of Horizons 2000. This means extending its timetable and being less ambitious in the long term. "Given the uncertainty of future funding", says its chairman Lodewijk Woltjer, an associate of the Observatoire de Haute Provence, southern France, "it no longer made sense to plan missions beyond 2010."

Missions already under way will adhere to their planned schedules. These include Cassini-Huygens, the NASA-led mission to Saturn due for launch in the autumn, a satellite dedicated to X-ray astronomy (1999 launch), Integral, the international gammaray astronomy laboratory (2001) and the cometary mission, Rosetta (2003).

The launch schedule for other planned missions will be extended. The cornerstone mission FIRST, the Far Infrared Space Tele-

\section{Mission made possible: the miracle mirror}

\section{IMAGE UNAVAILABLE FOR COPYRIGHT REASONS}

[WASHINGTON] A technician places glass over a mould during the casting of the first of two primary mirrors for the Large Binocular Telescope being built at Mount Graham, Arizona. The mirror was cast earlier this month using a new technique that involves pouring blocks of molten glass into a 'honeycomb' mould from a furnace at temperatures of $1,100^{\circ} \mathrm{C}$.

The process, known as 'spin casting', took 70 hours and was watched intermittently by 1,700 people. A further 12-week wait is in store before the mirror is cool enough for inspection; it will then have to be ground and polished. "Only then will we know whether the casting is a success," says Peter A. Strittmatter, director of the Steward Observatory at the University of Arizona.

The Large Binocular Telescope will consist of two primary mirrors, each 8.4 metres in diameter. Once completed, the Mount Graham instrument will have a resolving power which corresponds to that of a single 22.8-metre telescope, and lightgathering power equivalent to that of a single 11.8-metre instrument. scope, and the Planck Surveyor, previously known as COBRAS/SAMBA, which will examine the cosmic microwave background, will each be launched with relatively short delays between 2005 and 2007.

An additional medium-sized mission, earmarked for planetary science, will be launched later in the decade. But ESA's commitments now go no further. This means that the cornerstone mission to Mercury, originally due to launch in 2009 , is now on hold. So, too, are cornerstone missions dedicated to interferometry and the study of gravitational waves.

The advisory committee says that the advanced technologies required for these missions should continue to be developed in the expectation that they will be rescheduled in the future. Such technologies include ion propulsion, important for more efficient interplanetary travel. But the committee expresses concern that, even with the extended timetable, ESA's science programme will now have little money left to support such development studies.

Nor may there be sufficient money to make a significant contribution to two international programmes judged to be highly important for European space scientists. These are the missions to Mars and the Next Generation Space Telescope, the follow-up to the Hubble Space Telescope.

The committee recommends that the next medium-size mission, to be chosen in the next two or three years, should be at least in part dedicated to a Mars project. It asks the ESA science directorate to set up a task force to identify opportunities for ESA scientists to participate in NASA-led Mars missions, and to decide if use can be made of blueprints for instruments developed in Europe for the ill-fated Mars 96.

"But any participation in a mission to Mars will be disappointingly small," says Woltjer. The committee also asks ESA to set up a task force to follow the development by NASA of plans for the new telescope. It says the task force should identify opportunities for European astronomers to participate, for example by supplying the launcher or instruments, or by trading observing time with that on ESA's FIRST mission.

Steven Beckwith, director of the Max Planck Institute for Astrophysics in Heidelberg, says that the squeezed budgets which have forced the restructuring of the Horizons 2000 programme "will no doubt weaken European space science".

"It seems a shame that just when European space astronomy is coming into its own - and when the United States is investing heavily in new astronomy missions - its support is being cut back," he says.

Alison Abbott 